\title{
Molecular species delimitation in the primitively segmented spider genus Heptathela endemic to Japanese islands
}

Xin Xu ${ }^{\mathrm{a}, \mathrm{b},{ }^{*},+}$, Matjaž Kuntner ${ }^{\mathrm{b}, \mathrm{c}, \mathrm{d}, \mathrm{e},+}$, Jason E. Bond ${ }^{\mathrm{f}}$, Hirotsugu Ono ${ }^{\mathrm{g}}$, Simon Y. W. Ho ${ }^{\mathrm{h}}$, Fengxiang Liu ${ }^{\mathrm{b}}$, Long $\mathrm{Yu}^{\mathrm{b}}$, Daiqin $\mathrm{Li}^{\mathrm{i}}{ }^{\mathrm{i},}$

${ }^{a}$ College of Life Sciences, Hunan Normal University, Changsha, Hunan, China

${ }^{\mathrm{b} S t a t e}$ Key Laboratory of Biocatalysis and Enzyme Engineering, Centre for Behavioural Ecology and Evolution, School of Life Sciences, Hubei University, Wuhan, Hubei, China ${ }^{\mathrm{c}}$ Evolutionary Zoology Laboratory, Department of Organisms and Ecosystems Research, National Institute of Biology, Ljubljana, Slovenia

${ }^{\mathrm{d} E v o l u t i o n a r y}$ Zoology Laboratory, Biological Institute ZRC SAZU, Ljubljana, Slovenia ${ }^{\mathrm{e}}$ Department of Entomology, National Museum of Natural History, Smithsonian Institution, Washington, D.C., USA

${ }^{\mathrm{f}}$ Department of Entomology and Nematology, University of California at Davis, Davis, California, USA

${ }^{\mathrm{g}}$ Department of Zoology, National Museum of Nature and Science, 4-1-1 Amakubo, Tsukubashi, Ibaraki-ken, 305-0005, Japan

${ }^{\mathrm{h}}$ School of Life and Environmental Sciences, University of Sydney, Sydney, NSW, Australia

iDepartment of Biological Sciences, National University of Singapore, Singapore

${ }^{+}$Xin Xu and Matjaž Kuntner should be considered joint first authors

${ }^{*}$ Corresponding authors:

Xin $\mathrm{Xu}$, College of Life Sciences, Hunan Normal University, Changsha 410081, Hunan, China. Tel: +86-1857-0334-875; E-mail address: xuxin_09@163.com

Daiqin Li, Department of Biological Sciences, National University of Singapore, 14 Science Drive 4, Singapore 117543. Tel: +65-6516 4372; E-mail address: dbslidq@nus.edu.sg 


\begin{abstract}
Determining species boundaries forms an important foundation for biological research. However, the results of molecular species delimitation can vary with the data sets and methods that are used. Here we use a two-step approach to delimit species in the genus Heptathela, a group of primitively segmented trapdoor spiders that are endemic to Japanese islands. Morphological evidence suggests the existence of 19 species in the genus. We tested this initial species hypothesis by using six molecular species-delimitation methods to analyse 180 mitochondrial COI sequences of Heptathela sampled from across the known range of the genus. We then conducted a set of more focused analyses by sampling additional genetic markers from the subset of taxa that were inconsistently delimited by the single-locus analyses of mitochondrial DNA. Multilocus species delimitation was performed using two Bayesian approaches based on the multispecies coalescent. Our approach identified 20 putative species among the 180 sampled individuals of Heptathela. We suggest that our twostep approach provides an efficient strategy for delimiting species while minimizing costs and computational time.
\end{abstract}

Keywords: Liphistiidae; DNA barcode; species delimitation; multispecies coalescent; Bayes factor. 


\section{Introduction}

Species delimitation is an important process that underlies a wide range of biological questions (Agnarsson and Kuntner, 2007; Camargo and Sites, 2013). However, conflicts in species delimitation can be caused by differences in the application of species concepts (de Queiroz, 2007; Freudenstein et al., 2017) and in the choices of methods, models, and genetic markers (Carstens et al., 2013; Schlick-Steiner et al., 2010, 2014). Furthermore, there are differing views on the existence and importance of cryptic species (Fišer et al., 2018; Heethoff et al., 2018; Struck et al., 2018). This can pose challenges for the study of taxa that are well differentiated genetically but not morphologically (Bond et al., 2001; Leavitt et al., 2015). Resolving these conflicts is crucial for the advancement of integrative taxonomy.

Taxa inhabiting islands, mountains, and caves represent cases where lineages might be morphologically homogeneous but highly genetically structured. Island populations can experience morphological stasis as a result of shared selective pressure or niche conservatism (Gillespie and Roderick, 2002; Opatova and Arnedo, 2014). However, barriers to gene flow among isolated islands and among habitats within islands can lead to deep genetic structuring (Gillespie and Roderick, 2002; Opatova and Arnedo, 2014; Xu et al., 2016). The combination of morphological homogeneity with population genetic structuring can be seen in taxa with limited dispersal capability and high endemism (Derkarabetian et al., 2019; Hedin et al., 2015), thus presenting special challenges for species delimitation. Such conditions are seen in the Japanese archipelagos, which are among the most threatened biodiversity hotspots in the world (Crowe et al., 2006; Myers et al., 2000). These islands harbour diverse and largely endemic floras and faunas, including the primitively segmented trapdoor spiders (Araneae: Liphistiidae).

Liphistiidae is the only extant family in the suborder Mesothelae, an ancient and species-poor lineage of spiders that contains species with disjunct geographical distributions restricted to Southeast and East Asia (World Spider Catalog, 2020; Xu et al., 2015a, 2015b). The family has eight genera, each endemic to a particular geographical region. Like other arachnids (Derkarabetian and Hedin, 2014) and other trapdoor spiders (Bond et al., 2001; Satler et al., 2013), liphistiids often show limited dispersal ability and high habitat specificity (Haupt, 2003; Xu et al., 2015a). The biogeographic patterns in liphistiids make them an ideal system for studying speciation. However, species of liphistiids have historically been difficult to delimit because of their uniform, conservative morphology.

Liphistiid systematics and taxonomy are in need of revision, primarily due to insights from a recent series of molecular phylogenetic studies (Xu et al., 2015a, 2015b, 2015c, 2016, 2017). The efficacy of single-locus analyses based on the mitochondrial COI barcode region has been confirmed repeatedly by delimitation of species in the liphistiid genera Ganthela Xu \& Kuntner, 2015 and Ryuthela Haupt, 1983, enabling nomenclatural changes to be made (Xu et al., 2015c, 2017). Multilocus methods for species delimitation are expected to provide a more reliable identification of species boundaries, because they can collect information from multiple independently evolving loci. However, some studies have found that such methods detect population structure rather than species divergences, thus leading to oversplitting of species diversity in short-range endemic taxa (Chambers and Hillis, 2020; Hedin et al., 2015). In spite of this potential weakness, multilocus approaches can provide valuable information for guiding taxonomic decisions.

Here we focus on the liphistiid spider genus Heptathela Kishida, 1923 (Mesothelae, Liphistiidae), which is constrained to southwestern Japanese islands. It is known for its low vagility and inclination towards population structuring at small to moderate geographical scales (Xu et al., 2015a, 2015b, 2019). Prior to this study, there was taxonomic uncertainty in this genus. Twenty nominal Heptathela species are endemic to Kyushu and the northern and 
central Ryukyus (Tanikawa and Miyashita, 2014; World Spider Catalog, 2020; Xu et al., 2019), including 19 morphological species and one putative cryptic species. Four species are found in Kyushu, five in Amami, and 11 in Okinawa. Like other liphistiids, species of Heptathela lack clear diagnostic characters, are morphologically homogeneous among species, exhibit considerable intraspecific variation in female genital morphology, and/or are rare in collections (missing sexes or adult forms) (Xu et al., 2015c, 2017). As demonstrated for other liphistiid genera, Heptathela is potentially well suited to the application of an integrative approach to species delimitation.

In this study, we explore and validate species boundaries in Heptathela using molecular data via two steps. First, we analyse mitochondrial COI sequence data from 180 specimens of Heptathela to identify the lineages that are consistently delimited by a range of speciesdelimitation methods. Second, for any lineages that are not consistently delimited by these methods, we add mitochondrial and nuclear markers and apply multilocus, coalescent-based methods for species delimitation. The results of our analyses allow us to investigate the taxonomy of the genus using a molecular approach that efficiently identifies and investigates problematic lineages.

\section{Materials and methods}

\subsection{Taxon sampling and DNA sequencing}

We carried out three extensive collection trips to Japanese islands in December 2012, September 2013, and May 2014. Although our sampling was not exhaustive, we attempted to sample specimens from across the entire known range of Heptathela, from Kyushu to the central Ryukyu archipelago, based on the type locality information of each recorded Heptathela species (Fig. 1). We also searched previously unexplored areas. We made a great effort to apply the same strategy to each sampling location, but we often found no spiders because some species are very rare or have patchy distributions. Furthermore, some locations are urbanized habitats while others are inaccessible because of the lack of roads or the presence of military camps. Nevertheless, eight species were sampled from more than one population (3-14 individuals per population), and 12 species were collected from only one population with 2-10 individuals per species. Further details about sampling can be found in our taxonomic study (Xu et al., 2019).

We selected 180 specimens of Heptathela to form the ingroup for our analyses (Supplementary Table S1). In order to root the tree, we included two outgroup taxa from the liphistiid genus Ryuthela: R. nishihirai (Haupt, 1979) and R. shimojanai Xu, Liu, Ono, Chen, Kuntner, \& Li, 2017. These taxa are also endemic to Japanese islands (Xu et al., 2017).

From leg muscles of specimens preserved in absolute ethanol, we extracted genomic DNA using the Animal Genomic DNA Isolation Kit (Dingguo, Beijing, China). For all 182 samples, we amplified and sequenced the mitochondrial gene encoding cytochrome oxidase subunit 1 (COI) using the primer pair LCO1490/HCO2198 (Folmer et al., 1994). For 35 samples selected from the lineages of Heptathela that were not consistently resolved by different single-locus species-delimitation methods (see sections 2.2 and 3.1), we obtained sequence data from one additional mitochondrial gene (16S rRNA, 16S) and three nuclear markers (28S rRNA, 28S; internal transcribed spacer 2, ITS2; and histone 3, H3). DNA from these markers was amplified using the primer pairs 16Sar/16Sbr (Huber et al., 1993), 28SO/28S-C (Hedin and Maddison, 2001), ITS-5.8S/ITS-28S (White et al., 1990), and $\mathrm{H} 3 \mathrm{aF} / \mathrm{H} 3 \mathrm{aR}$ (Colgan et al., 1998), respectively. We followed previously reported standard PCR protocols for all genes (Xu et al., 2015a), then manually edited and aligned sequence data in Geneious Prime 2020 (https://www.geneious.com). Owing to a lack of variation in 
$28 S$ for the subset of 35 samples, we excluded this gene from our subsequent speciesdelimitation analyses.

\subsection{Single-locus species delimitation}

We inferred the evolutionary relationships among the sampled individuals of Heptathela using the COI data set. Our COI data set, comprising 676 bp sequences from 180 individuals of Heptathela, had 248 variable sites. For each of the three codon sites in the COI alignment, the GTR $+\mathrm{G}$ substitution model was selected using the Akaike information criterion in jModeltest v2.1.10 (Darriba et al. 2012). We chose not to consider models that allowed a proportion of invariable sites, owing to the shallow evolutionary timescale of Heptathela (Jia et al., 2014; Xu et al., 2016).

We performed Bayesian phylogenetic analyses of the COI alignment, partitioned by codon site, in MrBayes v3.2.1 (Ronquist et al., 2012). Posterior distributions were estimated by Markov chain Monte Carlo (MCMC) sampling. Samples were drawn every 2000 steps over a total of 20 million steps, with the initial $25 \%$ of samples discarded as burn-in. We performed the MCMC analysis in duplicate to check for convergence, and used the sump command to check for sufficient sampling. We also carried out a maximum-likelihood phylogenetic analysis of the COI alignment, partitioned by codon site, in IQ-TREE v1.6.12 (Nguyen et al., 2015). Node support was estimated using ultrafast bootstrapping with 1000 replicates (Hoang et al., 2018) and the Shimodaira-Hasegawa approximate likelihood-ratio test (SH-aLRT) with 1000 replicates (Guindon et al., 2010). The best-fitting substitution model for each of the three codon sites was selected using ModelFinder in IQ-TREE (Kalyaanamoorthy et al., 2017).

Our initial 19-species hypothesis for Heptathela is based on our accompanying morphological study of the genus (Xu et al., 2019). We tested this initial hypothesis by analysing mitochondrial $C O I$ sequence data with six commonly used single-locus speciesdelimitation methods. The first two methods were based on genetic distances: DNA barcoding gap (Barrett and Hebert, 2005) and automatic barcode gap discovery (ABGD; Puillandre et al., 2012). The remaining four methods were based on the inferred tree: generalized mixed Yule-coalescent (GMYC; Pons et al., 2006), the P ID(Liberal) method from the Species Delimitation plugin for Geneious (Masters et al., 2011), Bayesian Poisson tree processes (bPTP; Zhang et al., 2013), and multi-rate Poisson tree processes (mPTP; Kapli et al., 2017).

DNA barcoding gap is the most commonly used single-locus technique for species delimitation (Barrett and Hebert, 2005; Hamilton et al., 2014). It uses threshold values to differentiate between inter- and intraspecific divergences. We used MEGA X (Kumar et al., 2018 ) to calculate pairwise $\mathrm{K} 2 \mathrm{P}$ and $p$-distances, as well as mean intra- and interspecific $\mathrm{K} 2 \mathrm{P}$ and $p$-distances for each putative species. Unlike DNA barcoding gap analysis, ABGD does not require the sampled individuals to be assigned to putative species (Puillandre et al., 2012). ABGD detects the barcode gap based on the user-defined boundaries for intraspecific variability, then sorts the sampled individuals into candidate species with $p$-values. We performed ABGD analyses online (bioinfo.mnhn.fr/abi/public/abgd/), based on the K2P model, with a minimum intraspecific variability $P \min =0.001$, maximum intraspecific variability $P \max =0.1$, and minimum gap width $\mathrm{X}=1.5$.

We then used four tree-based methods for species delimitation. The P ID(Liberal) method, implemented in the Species Delimitation plugin in Geneious, tests different species delimitations that are defined a priori by the user (Masters et al., 2011). We used the phylogeny inferred in our Bayesian analysis as the guide tree to calculate the mean probability of the ratio of intra- to interspecific genetic distances for the initial 19-species hypothesis for Heptathela. 
The bPTP method, an updated version of the original PTP with Bayesian support values, assumes independent exponential distributions to model the branch lengths for speciation and for coalescence (Zhang et al., 2013). The newly developed mPTP, on the other hand, fits an independent exponential distribution to the branch lengths of each species (Kapli et al., 2017). We implemented bPTP on an online server (species.h-its.org/ptp/), using the maximum-likelihood phylogeny as the input tree. We ran the bPTP analysis for 500,000 steps, with a thinning of 500 and burn-in proportion of 0.1 . We performed species delimitations using likelihood and MCMC analyses on the maximum-likelihood tree in mPTP v0.2.4 (Kapli et al., 2017). In both analyses, we allowed differences in rates of coalescence among species and specified a minimum branch length of 0.0001. We ran MCMC analyses for 100 million steps, sampling every 10,000 , and discarded the first $10 \%$ of samples as burnin. Two independent MCMC analyses were run to check for convergence. Analyses starting from the maximum-likelihood species delimitation, random delimitation, and null delimitation all gave the same result.

The GMYC method uses likelihood to identify species boundaries by detecting the transition point between the speciation process and intraspecific lineage coalescence (Pons et al., 2006). We used the single-threshold model in the "splits" package (Ezard et al., 2009) for $\mathrm{R}$ v3.6.3 (R Core Team, 2020). The ultrametric guide tree was inferred using Bayesian analysis in BEAST v1.8.4 (Drummond et al., 2012). We compared two clock models (strict clock and relaxed lognormal clock; Drummond et al. 2006) and two tree priors (birth-death model with and without incomplete sampling) using marginal likelihoods estimated by stepping-stone sampling (Xie et al., 2011) with 100 path steps. The birth-death model provides a suitable tree prior for data sets with a mixture of intra- and interspecies sampling (Ritchie et al., 2017). To estimate the divergence times, we used a previous estimate of the COI substitution rate from other spider lineages (Bidegaray-Batista et al., 2014) to set a normal prior (mean 0.015 , standard deviation 0.001 ) on the substitution rate. The posterior distribution was estimated by MCMC sampling, with samples drawn every 5000 steps over a total of 50 million steps. We performed the analyses in duplicate to check for convergence and used Tracer to check for sufficient sampling.

\subsection{Multilocus species delimitation}

To investigate the lineages that were subject to inconsistent species delimitations across the six single-locus analyses described above (Fig. 1, red box), we performed multilocus species delimitation using two mitochondrial and two nuclear markers from 35 samples of Heptathela. The data set consisted of sequences from mitochondrial COI ( $n=35$, length 681 bp, 98 variable sites), mitochondrial $16 S$ ( $n=34$, length $491 \mathrm{bp}, 46$ variable sites), nuclear $H 3$ ( $n=34$, length $360 \mathrm{bp}, 17$ variable sites), and nuclear ITS2 ( $n=34$, length $344 \mathrm{bp}, 3$ variable sites). The best-fitting substitution models were HKY+G (16S and COI), F81 (ITS2), and $\mathrm{SYM}+\mathrm{G}(H 3)$. As above, we chose not to consider models that allowed a proportion of invariable sites. We concatenated the two mitochondrial markers and treated them as a single locus. We then concatenated the two nuclear markers because they had small numbers of variable sites.

We used two methods, Bayesian Phylogenetics and Phylogeography (BPP; Yang, 2015) and Bayes-factor delimitation (Grummer et al., 2014), to compare the four competing species hypotheses generated from our analyses of the COI data (see section 3.1). BPP has been shown to be effective in using information from gene trees to identify the boundaries between closely related species (Flouri et al., 2018; Yang and Rannala, 2017). Unguided Bayesian species delimitation was conducted in BPP v4.14 (Flouri et al., 2018) to explore different species-delimitation models and changes in the topology of the species tree (A11 analysis). 
For the priors on population sizes $(\theta)$ and divergence times $(\tau)$, we used inverse-Gamma priors with $\alpha=3$. The $\beta$ parameter was adjusted according to the mean estimate of nucleotide diversity for $\theta(\beta=0.2)$ and node height for $\tau(\beta=0.08)$. We performed the analyses using the reversible-jump MCMC algorithm $0(\varepsilon=2)$, and algorithm $1(\alpha=2, m=1)$, each run in duplicate to check for convergence. Analyses were run for 100,000 MCMC steps, with samples drawn every 5 steps and with the first $20 \%$ of samples discarded as burn-in.

Bayes-factor delimitation compares different species-tree models using their marginal likelihoods. We performed Bayes-factor delimitation in *BEAST (Heled and Drummond, 2010) using a strict molecular clock, a birth-death model for the species-tree prior, and piecewise-linear population sizes. Analyses were run for 50 million MCMC steps, with samples drawn every 5,000 steps and with the first $10 \%$ of samples treated as burn-in. We ran the analyses in duplicate to check for convergence and used Tracer to check for sufficient sampling. For each of the four competing species models, we estimated the marginal likelihood using stepping-stone sampling. Bayes factors (BF) were interpreted following the recommendations of Grummer et al. (2014), with $2 \ln \mathrm{BF}>10$ being considered as decisive support for a hypothesis. Where a comparison between two models was inconclusive, we favoured the simpler model.

\section{Results}

\subsection{Single-locus species delimitation}

Bayesian and maximum-likelihood methods inferred similar phylogenetic trees (Fig. 1, Supplementary Fig. S1). The initial species hypothesis (i.e., 19 species) was supported by three of the six species-delimitation methods that we compared: barcoding gap analysis, ABGD, and P ID(Liberal) (Fig. 1; Supplementary Tables S2 and S3). We detected a distinct gap between intra- and interspecific genetic distances for 19 hypothetical species of Heptathela, ranging from $4.2 \%$ to $5.7 \%$ for $\mathrm{K} 2 \mathrm{P}$ distances and from $4.0 \%$ to $5.4 \%$ for $p$ distances (Fig. 2). The smallest mean interspecific and largest mean intraspecific distances were $6.0 \% / 5.7 \%$ and $2.1 \% / 2.0 \%(\mathrm{~K} 2 \mathrm{P} / p$-distance $)$, respectively.

The initial species hypothesis was not supported by our analyses using GMYC, bPTP, and $\mathrm{mPTP}$, which delimited 21, 22, and 20 species, respectively. Nevertheless, 16 putative species were recognized by all six of the methods that we applied to the COI data set, and these are supported by morphological diagnosis in our taxonomic study (Xu et al., 2019). Based on these results, we derived four competing species hypotheses that we then tested using analyses of multilocus data (Fig. 1).

\subsection{Multilocus species delimitation}

Our BPP analysis of the multilocus data set yielded strong support for three or four species (posterior probability 1 ) or five species (posterior probability 0.97 ), with weaker support for six species (posterior probability 0.56) (Table 1). Bayes-factor delimitation showed that marginal likelihoods estimated using stepping-stone sampling provided the highest support for a model with six species, but found that this was not better than the fivespecies model $(2 \ln \mathrm{BF}=1.08)$ and only marginally better than the four-species model $(2 \ln \mathrm{BF}$ $=5.97$ ) (Table 2). In this case, we favour the four-species model because it is the simplest model that is not worse than the best-supported model. Taken together, our analyses using BPP and Bayes-factor delimitation support the four-species model.

\section{Discussion}


Our two-step approach to molecular species delimitation has yielded support for 20 putative species in the liphistiid spider genus Heptathela. The approach uses fast, single-locus delimitation methods to analyse an extensive taxon sample before applying more intensive multilocus methods to a chosen subset of the taxa. Our results confirm the taxonomic utility of the COI barcoding region by showing that a range of species-delimitation methods yield largely congruent estimates of species boundaries. Nevertheless, our approach also demonstrates the advantages of comparing the inferences from multiple methods for species delimitation.

Single-locus species-delimitation methods are routinely used to study taxa that are well differentiated genetically but not morphologically, such as liphistiid and mygalomorph spiders (Satler et al., 2013; Xu et al., 2015a, 2017). However, with the widespread acknowledgement of potential discordance between gene trees and the species tree due to incomplete lineage sorting (e.g., Harrington and Near, 2012; McGuire et al., 2007), species delimitation based on a single locus alone can be misleading. Therefore, multilocus methods based on the multispecies coalescent are expected to have a number of advantages. However, the field has not arrived at any single method that would be preferred for its reliability, costeffectiveness, and robustness.

Comparison of the performance of the six single-locus methods for species delimitation used in this study is not straightforward. Our previous studies on species limits in other liphistiid genera largely relied on the outcomes of DNA barcoding gap, ABGD, and P ID(Liberal) methods (Xu et al., 2015c, 2017). In the present study, all three of these methods support the initial, morphology-based hypothesis of 19 species. On the other hand, bPTP, mPTP, and GMYC support larger numbers of species. As in previous studies of simulated and empirical data (Luo et al., 2018; Tang et al., 2014), our results suggest that bPTP has a slight tendency to oversplit species. The GMYC method is also prone to delimiting larger numbers of species (Hamilton et al., 2014; Talavera et al., 2013).

An important challenge to species delimitation is the overestimation of species diversity, which can be caused by molecular approaches delimiting populations with geographic or genetic structure (Jackson et al., 2017; Sukumaran and Knowles, 2017). Furthermore, oversplitting can become worse when the amount of sequence data increases (Leaché et al., 2019). Bayes-factor delimitation is also prone to species oversplitting, especially if the same data are used to construct and test the species hypotheses (Grummer et al., 2014; Leaché et al., 2014). This problem of oversplitting is potentially heightened in short-range endemic taxa, such as liphistiid spiders, which often have high levels of geographic or population genetic structure (Chambers and Hillis, 2020; Hedin et al., 2015). However, our analyses of Heptathela show that both BPP and Bayes-factor delimitation support one of the more conservative species hypotheses that we considered.

Our species delimitations might have been negatively affected by the limitations on sampling. Many species in Heptathela are rare and have small geographical ranges, presenting considerable challenges to fieldwork and sample collection. Incomplete or geographically biased sampling can complicate molecular species delimitation (e.g., Ahrens et al., 2016; Carstens et al., 2013; Linck et al., 2019). For example, if all individuals from each species are collected from a single location, then intraspecific diversity will be underestimated, and barcode gaps will be overestimated. In this study, although 11 of the 16 species delimited using COI data were collected from a single population, they are consistent with morphological evidence (Xu et al., 2019). Barcode gaps are probably overestimated and will decrease with further sampling for each of these species. Nevertheless, our delimited species are likely to reflect the underlying evolutionary units in the genus Heptathela, based on current sampling. 
One can still argue that since the conflicting lineages are in a few locations where the geographic scale of sampling seems more 'fine grained' (i.e., multiple populations were sampled within a small geographic area), perhaps the species hypotheses generated from our analyses of $C O I$ sequence data would be less clear if this denser geographic sampling were applied throughout the genus Heptathela. Nevertheless, additional genetic data and increased geographic sampling of multiple populations will allow further testing of these species hypotheses.

In species of Heptathela from Kyushu and the Ryukyu archipelago, as in other liphistiid genera, females exhibit considerable intraspecific variation. In contrast, males are recognised with stable morphological characters, but they are very rarely collected (Haupt, 2003; Xu et al., 2015c). During our three extensive collection trips, we found both males and females for 12 species, but we found only females for the other eight species (Xu et al., 2019). For the reasons given above, a purely morphology-based taxonomic revision would be likely underestimate the true species diversity. Therefore, molecular species delimitation offers useful diagnostic evidence for the integrative taxonomy of liphistiids.

Eight Heptathela species were collected from more than one population, including two species (H. higoensis and H. kikuyai) that have wide distributions in Kyushu, and six species (H. kanenoi, H. uken, H. yanbaruensis, H. otoha, H. tokashiki, and H. crypta) with narrow distributions in the Ryukyu archipelago. Five of these eight species were identified by all six single-locus species-delimitation approaches, whereas the other three species were inconsistently delimited by the six methods. Nevertheless, these species delimitations were confirmed by the multilocus, coalescent-based methods. The remaining 12 species that were each collected from only a single population were also supported by single-locus methods, except $H$. unten was validated by multilocus species-delimitation approaches. The combined evidence from the geographical distributions and molecular species delimitation of Heptathela suggests that species diversity has not been overestimated. The Ryukyu archipelago has a higher diversity of species of Heptathela than Kyushu, despite the latter having a larger area.

In conclusion, our study has formalized an efficient two-step approach to molecular species delimitation by identifying problematic taxa and applying additional markers and analyses in a focused manner. Our analysis of primitively segmented trapdoor spiders of the genus Heptathela confirms the effectiveness of our approach, which can provide reliable species delineation. However, our results also demonstrate the need to evaluate multiple lines of evidence for an objective, repeatable test of species boundaries. 
bioRxiv preprint doi: https://doi.org/10.1101/812214; this version posted April 26, 2020. The copyright holder for this preprint (which was

not certified by peer review) is the author/funder, who has granted bioRxiv a license to display the preprint in perpetuity. It is made available under aCC-BY-NC-ND 4.0 International license.

\section{Acknowledgements}

We thank Arong Luo and Chengmin Shi for advice on data analyses. This study was supported by the grants from the National Science Foundation of China (NSFC-31601850; NSFC-31272324), the Hunan Provincial Natural Science Foundation of China (2017JJ3202), a bilateral China and Slovenia exchange project No. 12-8 from China, Bi-CN/18-20-022 from Slovenia, the Singapore Ministry of Education AcRF Tier 1 grant (R-154-000-A52-114), the Slovenian Research Agency (P1-0255, P1-10236, J1-672), and a visiting scholarship from China Scholarship Council to X.X. In part, analyses relied on the resources of the National Supercomputing Centre, Singapore (https://www.nscc.sg).

\section{Appendix Supplementary material}

DNA sequences are deposited in GenBank. DNA alignments and Tables S1-S3 as supplementary materials can be found online. 


\section{References}

Agnarsson, I., Kuntner, M., 2007. Taxonomy in a changing world: seeking solutions for a science in crisis. Syst. Biol. 56, 531-539. https://doi.org/10.1080/10635150701424546

Ahrens, D., Fujisawa, T., Krammer, H., Eberle, J., Fabrizi, S., Vogler, A.P., 2016. Rarity and incomplete sampling in DNA-based species delimitation. Syst. Biol. 65, 478-494. https://doi.org/10.1093/sysbio/syw002

Barrett, R.D.H., Hebert, P.D.N., 2005. Identifying spiders through DNA barcodes. Can. J. Zool. 83, 481-491. https://doi.org/10.1139/z05-024

Bidegaray-Batista, L., Ferrández, M.Á., Arnedo, M.A., 2014. Winter is coming: Miocene and Quaternary climatic shifts shaped the diversification of Western-Mediterranean Harpactocrates (Araneae, Dysderidae) spiders. Cladistics 30, 428-446. https://doi.org/10.1111/cla.12054

Bond, J.E., Hedin, M.C., Ramirez, M.G., Opell, B.D., 2001. Deep molecular divergence in the absence of morphological and ecological change in the Californian coastal dune endemic trapdoor spider Aptostichus simus. Mol. Ecol. 10, 899-910. https://doi.org/10.1046/j.1365-294X.2001.01233.x

Camargo, A., Sites, J., 2013. Species delimitation: a decade after the renaissance, in: Pavlinov, I.Y. (Ed.), The Species Problem - Ongoing Issues. InTech, New York, pp. 225-247. https://doi.org/10.5772/52664

Carstens, B.C., Pelletier, T.A., Reid, N.M., Satler, J.D., 2013. How to fail at species delimitation. Mol. Ecol. 22, 4369-4383. https://doi.org/10.1111/mec.12413

Chambers, E.A., Hillis, D.M., 2020. The multispecies coalescent over-splits species in the case of geographically widespread taxa. Syst. Biol. 69, 184-193. https://doi.org/10.1093/sysbio/syz042

Colgan, D.J., McLauchlan, A., Wilson, G.D.F., Livingston, S.P., Edgecombe, G.D., Macaranas, J., Cassis, G., Gray, M.R., 1998. Histone H3 and U2 snRNA DNA sequences and arthropod molecular evolution. Aust. J. Zool. 46, 419-437. https://doi.org/10.1071/ZO98048

Crowe, T.M., Bowie, R.C., Bloomer, P., Mandiwana, T.G., Hedderson, T.A.J., Randi, E., Pereira, S.L., Wakeling, J., 2006. Phylogenetics, biogeography and classification of, and character evolution in, gamebirds (Aves: Galliformes): effects of character exclusion, data partitioning and missing data. Cladistics 22, 495-532.

Darriba, D., Taboada, G.L., Doallo, R., Posada, D., 2012. jModelTest 2: more models, new heuristics and parallel computing. Nat. Methods 9: 772.

de Queiroz, K., 2007. Species concepts and species delimitation. Syst. Biol. 56, 879-886. https://doi.org/10.1080/10635150701701083

Derkarabetian, S., Castillo, S., Koo, P.K., Ovchinnikov, S., Hedin, M., 2019. A demonstration of unsupervised machine learning in species delimitation. Mol. Phylogenet. Evol. 139, 106562. https://doi.org/10.1016/j.ympev.2019.106562

Derkarabetian, S., Hedin, M., 2014. Integrative taxonomy and species delimitation in harvestmen: a revision of the western North American genus Sclerobunus (Opiliones: Laniatores: Travunioidea). PLOS ONE 9, e112001. https://doi.org/10.1371/journal.pone.0104982

Drummond, A.J., Ho, S.Y.W., Phillips, M.J., Rambaut, A., 2006. Relaxed phylogenetics and dating with confidence. PLOS Biol. 4, e88. https://doi.org/10.1371/journal.pbio.0040088

Drummond, A.J., Suchard, M.A., Xie, D., Rambaut, A., 2012. Bayesian phylogenetics with BEAUti and the BEAST 1.7. Mol. Biol. Evol. 29, 1969-1973. https://doi.org/10.1093/molbev/mss075

Ezard, T., Fujisawa, T., Barraclough, T.G., 2009. SPLITS: Species' limits by threshold 
statistics. R Package Version 1.0-11.

Fišer, C., Robinson, C.T., Malard, F., 2018. Cryptic species as a window into the paradigm shift of the species concept. Mol. Ecol. 27, 613-635. https://doi.org/10.1111/mec.14486

Flouri, T., Jiao, X., Rannala, B., Yang, Z., 2018. Species tree inference with BPP using genomic sequences and the multispecies coalescent. Mol. Biol. Evol. 35, 2585-2593. https://doi.org/10.1093/molbev/msy147

Folmer, O., Black, M., Hoeh, W., Lutz, R., Vrijenhoek, R., 1994. DNA primers for amplification of mitochondrial cytochrome c oxidase subunit I from diverse metazoan invertebrates. Mol. Mar. Biol. Biotechnol. 3, 294-299.

Freudenstein, J. V., Broe, M.B., Folk, R.A., Sinn, B.T., 2017. Biodiversity and the species concept - lineages are not enough. Syst. Biol. 66, 644-656. https://doi.org/10.1093/sysbio/syw098

Gillespie, R.G., Roderick, G.K., 2002. Arthopods on islands: colonisation, speciation, and conservation. Annu. Rev. Entomol. 47, 595-632.

Grummer, J.A., Bryson, R.W., Reeder, T.W., 2014. Species delimitation using bayes factors: simulations and application to the Sceloporus scalaris species group (Squamata: Phrynosomatidae). Syst. Biol. 63, 119-133. https://doi.org/10.1093/sysbio/syt069

Guindon, S., Dufayard, J.F., Lefort, V., Anisimova, M., Hordijk, W., Gascuel, O., 2011. New algorithms and methods to estimate maximum-likelihood phylogenies: assessing the performance of PhyML 3.0. Syst Biol. 59, 307-321. https://doi.org/10.1093/sysbio/syq010.

Hamilton, C. a, Hendrixson, B.E., Brewer, M.S., Bond, J.E., 2014. An evaluation of sampling effects on multiple DNA barcoding methods leads to an integrative approach for delimiting species: a case study of the North American tarantula genus Aphonopelma (Araneae, Mygalomorphae, Theraphosidae). Mol. Phylogenet. Evol. 71, 79-93. https://doi.org/10.1016/j.ympev.2013.11.007

Harrington, R.C., Near, T.J., 2012. Phylogenetic and coalescent strategies of species delimitation in snubnose darters (Percidae: Etheostoma). Syst. Biol. 61, 63-79. https://doi.org/10.1093/sysbio/syr077

Haupt, J., 2003. The Mesothelae - a monograph of an exceptional group of spiders (Araneae: Mesothelae) (Morphology, behaviour, ecology, taxonomy, distribution and phylogeny). Zoologica 154, 1-102.

Hedin, M., Carlson, D., Coyle, F., 2015. Sky island diversification meets the multispecies coalescent - divergence in the spruce-fir moss spider (Microhexura montivaga, Araneae, Mygalomorphae) on the highest peaks of southern Appalachia. Mol. Ecol. 24, 34673484. https://doi.org/10.1111/mec.13248

Hedin, M.C., Maddison, W.P., 2001. Phylogenetic utility and evidence for multiple copies of elongation factor- $1 \alpha$ in the spider genus Habronattus (Araneae: Salticidae). Mol. Biol. Evol. 18, 1512-1521. https://doi.org/10.1093/oxfordjournals.molbev.a003937

Heethoff, M., Bendiksby, M., Birkeland, S., Cerca, J., Gusarov, V.I., Kistenich, S., Larsson, K., Liow, L.H., Nowak, M.D., Stedje, B., Bachmann, L., 2018. Cryptic species conceptual or terminological chaos? A response to Struck et al. Trends Ecol. Evol. 33, 310. https://doi.org/10.1016/j.tree.2018.02.008

Heled, J., Drummond, A.J., 2010. Bayesian inference of species trees from multilocus data. Mol Biol Evol 27, 570-580. https://doi.org/10.1093/molbev/msp274

Hoang, D.T., Chernomor, O., von Haeseler, A., Minh, B.Q., Vinh, L.S., 2018. UFBoot2: Improving the ultrafast bootstrap approximation. Mol. Biol. Evol. 35, 518-522. https://doi.org/10.1093/molbev/msx281

Huber, K., Haider, T., Mueller, M., 1993. DNA sequence data indicates the polyphyly of the family Ctenidae (Araneae). J. Arachnol. 21, 194-201. https://doi.org/10.2307/3705482 
Jackson, N.D., Carstens, B.C., Morales, A.E., O’Meara, B.C., 2017. Species delimitation with gene flow. Syst. Biol. 66, 799-812. https://doi.org/10.1093/sysbio/syw117

Jia, F., Lo, N., Ho, S.Y.W., 2014. The impact of modelling rate heterogeneity among sites on phylogenetic estimates of intraspecific rates and timescales. PLOS ONE 9, e95722. https://doi.org/10.1371/journal.pone.0095722

Kalyaanamoorthy, S., Minh, B.Q., Wong, T.K.F., von Haeseler, A., Jermiin, L.S., 2017, ModelFinder: Fast model selection for accurate phylogenetic estimates. Nat. Methods 14, 587-589. https://doi.org/10.1038/nmeth.4285

Kapli, P., Lutteropp, S., Zhang, J., Kobert, K., Pavlidis, P., Stamatakis, A., Flouri, T., 2017. Multi-rate poisson tree processes for single-locus species delimitation under maximum likelihood and Markov Chain Monte Carlo. Bioinformatics 33, 1630-1638. https://doi.org/10.1093/bioinformatics/btx025

Kumar, S., Stecher, G., Li, M., C. Knyaz, Tamura, K., 2018. MEGA X: molecular evolutionary genetics analysis across computing platforms. Mol Biol Evol. 35, 15471549. https://doi.org/10.1093/molbev/msy096

Leaché, A.D., Fujita, M.K., Minin, V.N., Bouckaert, R.R., 2014. Species delimitation using genome-wide SNP data. Syst. Biol. 63, 534-542. https://doi.org/10.1093/sysbio/syu018

Leaché, A.D., Zhu, T., Rannala, B., Yang, Z., 2019. The spectre of too many species. Syst. Biol. 68, 168-181. https://doi.org/10.1093/sysbio/syy051

Leavitt, D.H., Starrett, J., Westphal, M.F., Hedin, M., 2015. Multilocus sequence data reveal dozens of putative cryptic species in a radiation of endemic Californian mygalomorph spiders (Araneae, Mygalomorphae, Nemesiidae). Mol. Phylogenet. Evol. 91, 56-67. https://doi.org/10.1016/j.ympev.2015.05.016

Linck, E., Epperly, K., Van Els, P., Spellman, G. M., Bryson, R. W., McCormack, J. E., ... Klicka, J., 2019. Dense geographic and genomic sampling reveals paraphyly and a cryptic lineage in a classic sibling species complex. Syst. Biol. 68, 956-966. https://doi.org/10.1093/sysbio/syz027

Luo, A., Ling, C., Ho, S.Y.W., Zhu, C.-D., 2018. Comparison of methods for molecular species delimitation across a range of speciation scenarios. Syst. Biol. 67, 830-846. https://doi.org/10.1093/sysbio/syy011

Masters, B.C., Fan, V., Ross, H.A., 2011. Species Delimitation - a Geneious plugin for the exploration of species boundaries. Mol. Ecol. Resour. 11, 154-157. https://doi.org/10.1111/j.1755-0998.2010.02896.x

McGuire, J.A., Linkem, C.W., Koo, M.S., Hutchison, D.W., Lappin, A.K., Orange, D.I., Lemos-Espinal, J., Riddle, B.R., Jaeger, J.R., 2007. Mitochondrial introgression and incomplete lineage sorting through space and time: phylogenetics of crotaphytid lizards. Evolution 61, 2879-2897. https://doi.org/10.1111/j.1558-5646.2007.00239.x

Myers, N., Mittermeier, R.A., Mittermeier, C.C., da Fonseca, G.A., Kent, J., 2000. Biodiversity hotspots for conservation priorities. Nature 403, 853-858.

Nguyen, L.T., Schmidt, H.A., von Haeseler, A., Minh, B.Q., 2015. IQ-TREE: A fast and effective stochastic algorithm for estimating maximum likelihood phylogenies. Mol. Biol. Evol. 32, 268-274. https://doi.org/10.1093/molbev/msu300

Opatova, V., Arnedo, M.A., 2014. Spiders on a hot volcanic roof: colonisation pathways and phylogeography of the Canary Islands endemic trap-door spider Titanidiops canariensis (Araneae, Idiopidae). PLOS ONE 9, e115078. https://doi.org/10.1371/journal.pone.0115078

Pons, J., Barraclough, T., Gomez-Zurita, J., Cardoso, A., Duran, D., Hazell, S., Kamoun, S., Sumlin, W., Vogler, A., 2006. Sequence-based species delimitation for the DNA taxonomy of undescribed insects. Syst. Biol. 55, 595-609. https://doi.org/10.1080/10635150600852011 
Puillandre, N., Lambert, a, Brouillet, S., Achaz, G., 2012. ABGD, Automatic Barcode Gap Discovery for primary species delimitation. Mol. Ecol. 21, 1864-1877. https://doi.org/10.1111/j.1365-294X.2011.05239.x

R Core Team (2020). R: A language and environment for statistical computing. http://www.R-project.org/.

Ritchie, A.M., Lo, N., Ho, S.Y.W., 2016. The impact of the tree prior on molecular dating of data sets containing a mixture of inter- and intraspecies sampling. Syst. Biol. 66, 413425. https://doi.org/10.1093/sysbio/syw095

Ronquist, F., Teslenko, M., van der Mark, P., Ayres, D.L., Darling, A., Höhna, S., Larget, B., Liu, L., Suchard, M.A., Huelsenbeck, J.P., 2012. MrBayes 3.2: efficient Bayesian phylogenetic inference and model choice across a large model space. Syst. Biol. 61, 539-542. https://doi.org/10.1093/sysbio/sys029

Satler, J.D., Carstens, B.C., Hedin, M., 2013. Multilocus species delimitation in a complex of morphologically conserved trapdoor spiders (Mygalomorphae, Antrodiaetidae, Aliatypus). Syst. Biol. 62, 805-823. https://doi.org/10.1093/sysbio/syt041

Schlick-Steiner, B.C., Arthofer, W., Steiner, F.M., 2014. Take up the challenge! Opportunities for evolution research from resolving conflict in integrative taxonomy. Mol. Ecol. 23, 4192-4194. https://doi.org/10.1111/mec.12868

Schlick-Steiner, B.C., Steiner, F.M., Seifert, B., Stauffer, C., Christian, E., Crozier, R.H., 2010. Integrative taxonomy: a multisource approach to exploring biodiversity. Annu. Rev. Entomol. 55, 421-438. https://doi.org/10.1146/annurev-ento-112408-085432

Struck, T.H., Feder, J.L., Bendiksby, M., Birkeland, S., Cerca, J., Gusarov, V.I., Kistenich, S., Larsson, K., Liow, L.H., Nowak, M.D., Stedje, B., Bachmann, L., Dimitrov, D., 2018. Finding evolutionary processes hidden in cryptic species. Trends Ecol. Evol. 33, 153163. https://doi.org/10.1016/j.tree.2017.11.007

Sukumaran, J., Knowles, L.L., 2017. Multispecies coalescent delimits structure, not species. Proc. Natl Acad. Sci. U.S.A. 114, 1607-1612. https://doi.org/10.1073/pnas.1607921114

Talavera, G., Lukhtanov, V.A., Rieppel, L., Pierce, N.E., Vila, R., 2013. In the shadow of phylogenetic uncertainty: the recent diversification of Lysandra butterflies through chromosomal change. Mol. Phylogenet. Evol. 69, 469-478. https://doi.org/10.1016/j.ympev.2013.08.004

Tang, C.Q., Humphreys, A.M., Fontaneto, D., Barraclough, T.G., 2014. Effects of phylogenetic reconstruction method on the robustness of species delimitation using single-locus data. Methods Ecol. Evol. 5, 1086-1094. https://doi.org/10.1111/2041210X.12246

Tanikawa, A., Miyashita, T., 2014. Discovery of a cryptic species of Heptathela from the northernmost part of Okinawajima Is., Southwest Japan, as revealed by mitochondrial and nuclear DNA. Acta Arachnol. 63, 65-72. https://doi.org/10.2476/asjaa.63.65

White, T.J., Bruns, T., Lee, S., Taylor, J., 1990. Amplification and direct sequencing of fungal ribosomal RNA genes for phylogenetics, in: PCR Protocols. Elsevier, pp. 315322. https://doi.org/10.1016/B978-0-12-372180-8.50042-1

World Spider Catalog, 2020. World Spider Catalog. Version 21.0. Natural History Museum Bern, online at http://wsc.nmbe.ch (accessed on 08 March 2020)

Xie, W., Lewis, P.O., Fan, Y., Kuo, L., Chen, M.H., 2011. Improving marginal likelihood estimation for Bayesian phylogenetic model selection. Syst. Biol. 60, 150-160. https://doi.org/10.1093/sysbio/syq085

Xu, X., Liu, F.X., Cheng, R.C., Chen, J., Xu, X., Zhang, Z., Ono, H., Pham, D.S., NormaRashid, Y., Arnedo, M., Kuntner, M., Li, D., 2015a. Extant primitively segmented spiders have recently diversified from an ancient lineage. Proc. Biol. Sci. 282, 20142486.

Xu, X., Liu, F.X., Chen, J., Ono, H., Li, D., Kuntner, M., 2015b. A genus-level taxonomic 
review of primitively segmented spiders (Mesothelae: Liphistiidae). ZooKeys 488, 121151. https://doi.org/10.3897/zookeys.488.8726

Xu, X., Liu, F., Chen, J., Li, D., Kuntner, M., 2015c. Integrative taxonomy of the primitively segmented spider genus Ganthela (Araneae: Mesothelae: Liphistiidae): DNA barcoding gap agrees with morphology. Zool. J. Linn. Soc. 175, 288-306.

https://doi.org/10.1111/zoj.12280

Xu, X., Liu, F., Chen, J., Ono, H., Agnarsson, I., Li, D., Kuntner, M., 2016. Pre-Pleistocene geological events shaping diversification and distribution of primitively segmented spiders on East Asian margins. J. Biogeogr. 43, 1004-1019. https://doi.org/10.1111/jbi.12687

Xu, X., Liu, F., Ono, H., Chen, J., Kuntner, M., Li, D., 2017. Targeted sampling in Ryukyus facilitates species delimitation of the primitively segmented spider genus Ryuthela (Araneae: Mesothelae: Liphistiidae). Zool. J. Linn. Soc. 181, 867-909. https://doi.org/10.1093/zoolinnean/zlx024

Xu X., Ono H., Kuntner M., Liu F., Li D. 2019. A taxonomic monograph of the liphistiid spider genus Heptathela, endemic to Japanese islands. ZooKeys 888, 1-50. https://doi.org/10.3897/zookeys.888.34494

Yang, Z., 2015. The BPP program for species tree estimation and species delimitation. Curr. Zool. 61, 854-865. https://doi.org/10.1093/czoolo/61.5.854

Yang, Z., Rannala, B., 2017. Bayesian species identification under the multispecies coalescent provides significant improvements to DNA barcoding analyses. Mol. Ecol. 26, 30283036. https://doi.org/10.1111/mec.14093

Zhang, J., Kapli, P., Pavlidis, P., Stamatakis, A., 2013. A general species delimitation method with applications to phylogenetic placements. Bioinformatics 29, 2869-2876.

https://doi.org/10.1093/bioinformatics/btt499 
Table 1. Posterior probabilities from Bayesian analyses of multilocus data in BPP analyses, computed under four competing species hypotheses for the genus Heptathela

\begin{tabular}{|c|c|c|c|c|c|}
\hline \multirow{3}{*}{ Hypothesis } & \multirow{3}{*}{ Delimited species } & \multicolumn{4}{|c|}{ Posterior probability } \\
\hline & & \multicolumn{2}{|c|}{ rjМСМC $(0,2)$} & \multicolumn{2}{|c|}{$\operatorname{rjMCMC~}(1,2,1)$} \\
\hline & & run 1 & run2 & run 1 & run2 \\
\hline \multirow[t]{4}{*}{3 species } & & 1.00 & 1.00 & 1.00 & 1.00 \\
\hline & otoha & 1.00 & 1.00 & 1.00 & 1.00 \\
\hline & crypta + unten & 1.00 & 1.00 & 1.00 & 1.00 \\
\hline & yanbarunesis & 1.00 & 1.00 & 1.00 & 1.00 \\
\hline \multirow[t]{5}{*}{4 species } & & 1.00 & 1.00 & 1.00 & 1.00 \\
\hline & otoha & 1.00 & 1.00 & 1.00 & 1.00 \\
\hline & crypta & 1.00 & 1.00 & 1.00 & 1.00 \\
\hline & unten & 1.00 & 1.00 & 1.00 & 1.00 \\
\hline & yanbarunesis & 1.00 & 1.00 & 1.00 & 1.00 \\
\hline \multirow[t]{6}{*}{5 species } & & 0.97 & 0.97 & 0.97 & 0.97 \\
\hline & otoha & 0.98 & 0.97 & 0.98 & 0.98 \\
\hline & crypta & 1.00 & 1.00 & 1.00 & 1.00 \\
\hline & unten & 1.00 & 1.00 & 1.00 & 1.00 \\
\hline & yanbarunesis 1 & 0.97 & 0.97 & 0.97 & 0.97 \\
\hline & yanbarunesis 2 & 0.99 & 0.99 & 0.99 & 0.99 \\
\hline \multirow[t]{7}{*}{6 species } & & 0.55 & 0.55 & 0.56 & 0.55 \\
\hline & otoha 1 & 0.64 & 0.63 & 0.64 & 0.63 \\
\hline & otoha 2 & 0.58 & 0.57 & 0.58 & 0.57 \\
\hline & crypta & 0.99 & 1.00 & 1.00 & 1.00 \\
\hline & unten & 0.99 & 0.99 & 0.99 & 1.00 \\
\hline & yanbarunesis 1 & 0.87 & 0.88 & 0.87 & 0.88 \\
\hline & yanbarunesis 2 & 0.99 & 0.99 & 0.99 & 0.99 \\
\hline
\end{tabular}


bioRxiv preprint doi: https://doi.org/10.1101/812214; this version posted April 26, 2020. The copyright holder for this preprint (which was

not certified by peer review) is the author/funder, who has granted bioRxiv a license to display the preprint in perpetuity. It is made available under aCC-BY-NC-ND 4.0 International license.

Table 2. Marginal likelihoods of four competing species hypotheses for the genus Heptathela, computed from a multilocus data set. The log marginal likelihoods are ranked from highest to lowest; the Bayes factor (BF) is calculated using $2 \times$ (marginal likelihood of species model 1 - marginal likelihood of species model 2), with $2 \ln \mathrm{BF}>10$ being considered as decisive support for species model 1 .

\begin{tabular}{lccc}
\hline Species model & $\begin{array}{c}\text { Log marginal } \\
\text { likelihood }\end{array}$ & Rank & 2/nBF \\
\hline 3 species & -4180.17 & 4 & 40.25 \\
4 species & -4163.03 & 3 & 5.97 \\
5 species & -4160.59 & 2 & 1.08 \\
6 species & -4160.05 & 1 & \\
\hline
\end{tabular}



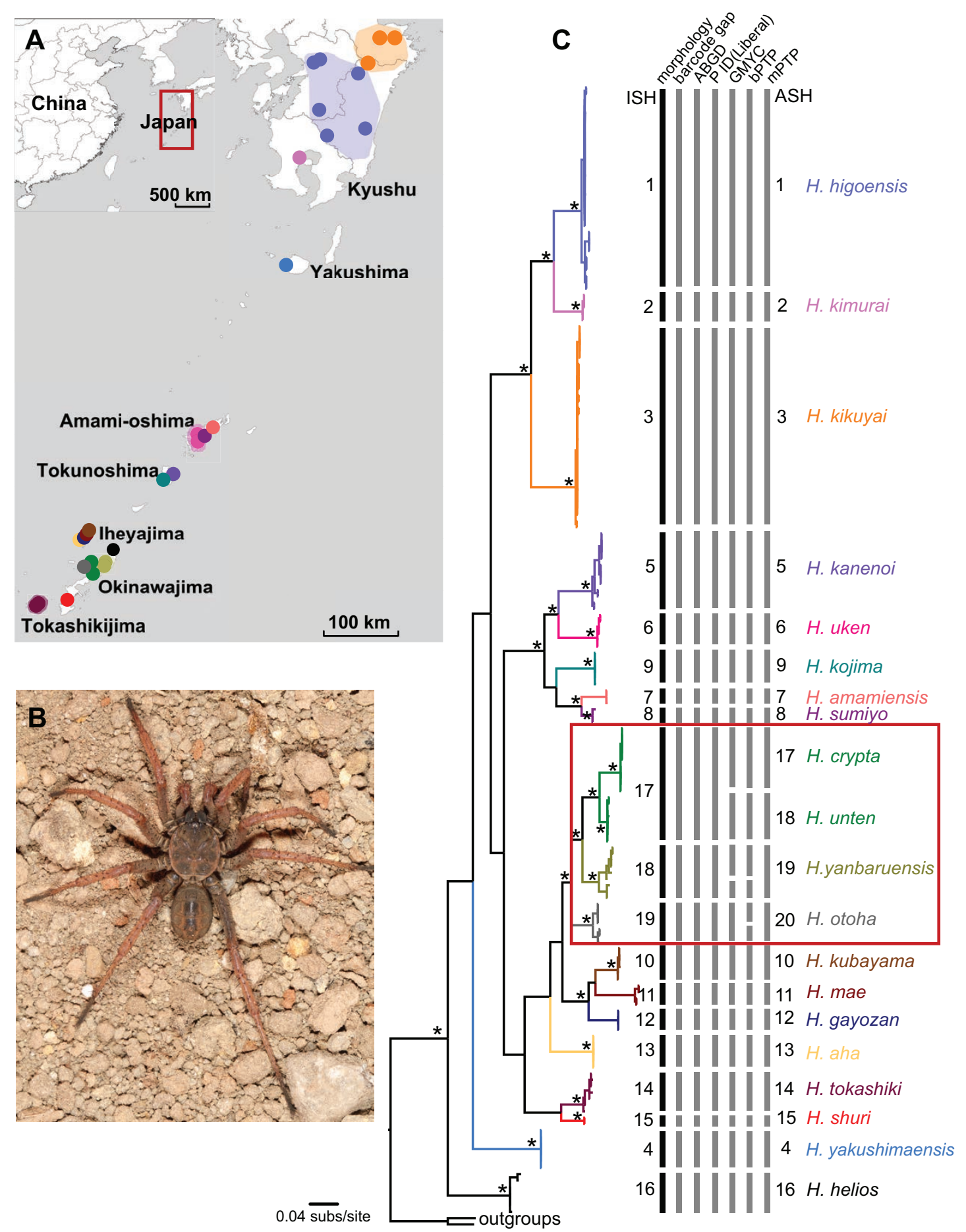

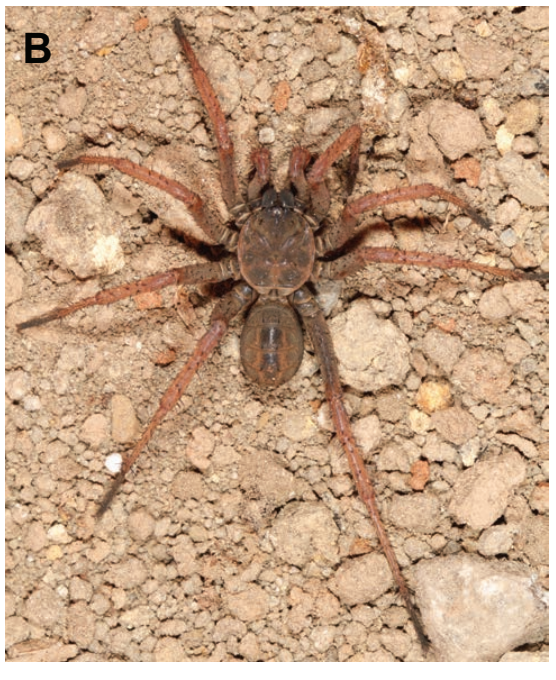

$0.04 \overline{\text { subs }} /$ site
(16. hes

Fig. 1. (A) Map showing all sampling localities for species of Heptathela on Kyushu and the Ryukyu archipelago (for a more detailed map, see Xu et al., 2019). (B) Heptathela kojima, male (body length: $10.40 \mathrm{~mm}$ ), from Amami-oshima, showing the general somatic morphology. (C) Bayesian tree for 180 samples of Heptathela, rooted using two outgroup taxa, Ryuthela nishihirai and R. shimojanai. Asterisks indicate strongly supported nodes (posterior probability $>0.95$, likelihood bootstrap support $>70 \%$ ). Clades are colour coded to match the map. ISH: initial species hypothesis (19 species), based on morphology and geographic information. ASH: alternative species hypothesis. Vertical bars indicate species delimitations based on morphology and on six different single-locus species delimitation methods. The red box indicates the lineages for which there was disagreement among the six species-delimitation methods. 

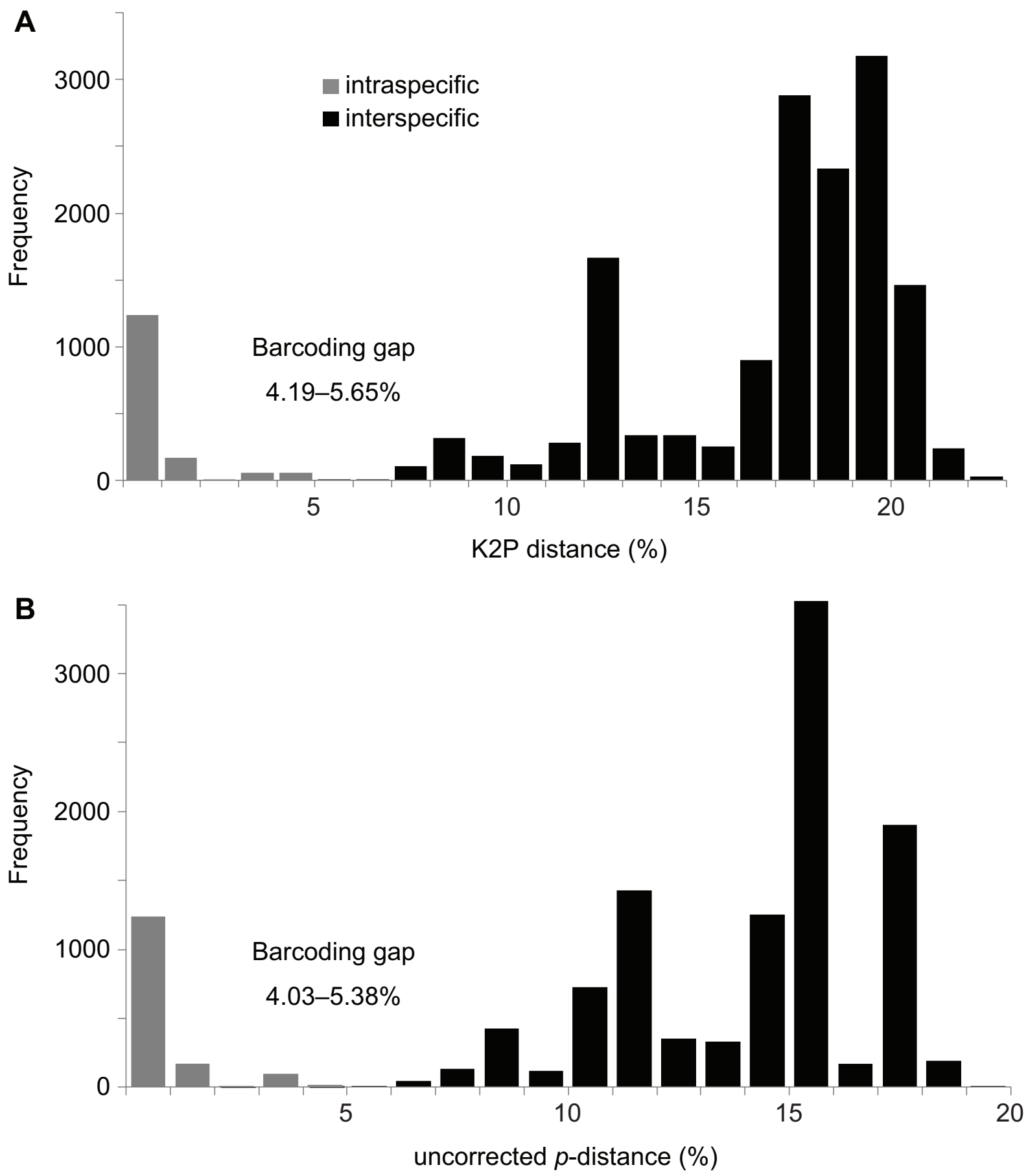

Fig. 2. DNA barcoding gap for COI sequences from 19 putative species of Heptathela, based on (A) K2P distances and (B) $p$-distances. 